\title{
Esclerosis Tuberosa: presentación de un caso clínico
}

\author{
Tuberous Sclerosis: a case report
}

\author{
Susana Rivera García, Ana Arévalo Gómez, Fernando de la Iglesia Martínez \\ Unidad de Enfermedades Minoritarias. Servicio de Medicina Interna. Complejo Hospitalario Universitario A Coruña
}

\section{RESUMEN}

La Esclerosis Tuberosa (ET) ó Complejo Esclerosis Tuberosa es un trastorno genético autosómico dominante que afecta a niños y adultos. Se debe a la ausencia parcial o total de la expresión de los genes TSC1 (hamartina) o TSC2 (tuberina), lo que genera disfunción orgánica por crecimiento de hamartomas en sistema nervioso central, riñón, corazón, pulmón y piel. Un seguimiento protocolizado nos ayuda a confirmar el diagnóstico así como a identificar precozmente complicaciones clínicamente significativas. Nos permite además identificar portadores de mutaciones entre los familiares y realizar eficazmente labores de consejo genético.

Palabras clave: Esclerosis tuberosa, placas de Shagreen, angiofibromas, epilepsia.

\section{INTRODUCCIÓN}

La Esclerosis Tuberosa (ET) es una enfermedad multisistémica cuyas manifestaciones clínicas son muy variables. Por este motivo, los pacientes con esta patología requieren un seguimiento multidisciplinar a lo largo de su vida que implica la realización de numerosos controles periódicos.

La baja sensibilización con esta patología, junto con la ausencia de un especialista de referencia para estos pacientes en la edad adulta, hacen que en un gran número de hospitales estos pacientes se encuentren en muchos casos sin seguir.

En el año 2017 se creó en nuestro hospital una consulta monográfica de Esclerosis Tuberosa para facilitar el correcto estudio, seguimiento y tratamiento de estos pacientes y sus familiares, así como la transición de los mismos de la edad pediátrica a la edad adulta.

La consulta monográfica está centralizada y coordinada en el servicio de Medicina Interna que se encarga de la evaluación integral con el apoyo de un comité multidisciplinar en el que están representados profesionales de todas las especialidades involucradas en esta patología.

Para su estudio y seguimiento se sigue un protocolo elaborado por el comité multidisciplinar.

A continuación se presenta uno de los casos clínicos a seguimiento en la consulta.

\section{CASO CLÍNICO}

Se trata de un varón de 55 años con diagnóstico de esclerosis tuberosa remitido a la consulta para seguimiento multidisciplinar. Sus padres viven y están sanos y uno de sus tres hijos presenta la misma enfermedad. Los otros dos

\begin{abstract}
Tuberous Sclerosis (TS) or Tuberous Sclerosis Complex is an autosomal dominant genetic disorder that affects children and adults. It is due to the partial or total absence of the expression of the TSC1 (hamartin) or TSC2 (tuberin) genes, which generates organic dysfunction due to the growth of hamartomas in the central nervous system, kidney, heart, lung and skin. A protocolized follow-up helps us to confirm the diagnosis as well as to promptly detect clinically significant complications. It also allows us to identify carriers of mutations among relatives and effectively carry out genetic counseling tasks.
\end{abstract}

Keywords: Tuberous sclerosis; Shagreen patches; angiofibroma;.epilepsy.

hijos no han sido estudiados y están asintomáticos. No tiene realizado estudio genético.

El paciente presenta epilepsia desde la infancia a seguimiento por neurología sin crisis en los últimos años y a tratamiento con levetiracetam. En la tomografia axial computerizada (TAC) cerebral (imagen 1) presenta múltiples calcificaciones subependimarias en ambos ventrículos laterales (hamartomas) y túber cortical en el giro frontal superior derecho y en las folias de ambos hemisferios cerebelosos. A nivel renal presenta múltiples angiomiolipomas bilaterales, el de mayor tamaño en el riñón derecho de 3,6 cm x 4 $\mathrm{cm}$ y múltiples quistes corticales simples y pielocaliciales bilaterales (imagen 2). El tamaño de los angiomiolipomas ha permanecido estable durante los últimos años. A nivel cutáneo presenta lesiones en confetti, placa de Shagreen (imagen 3) y cinco fibromas intraorales (imagen 4). No presenta afectación oftálmica ni clínica respiratoria.

Este paciente cumple criterios clínicos para esclerosis tuberosa definida: tres criterios mayores que son la placa de shagreen, los angiomiolipomas renales y la afectación cerebral con hamartomas y tubers corticales y tres criterios menores, las lesiones cutáneas en confetti, los quistes renales múltiples y los fibromas intraorales.

El seguimiento clínico del paciente se realiza de forma anual y multidisciplinar por Urología, Neurología y Medicina Interna siguiendo un protocolo realizado en nuestro centro.

Dado la presencia de un angiomiolipoma de $4 \mathrm{~cm}$ se valoró el inicio de tratamiento con everolimus, un fármaco inhibidor de la vía mTOR, pero el paciente prefiere demorarlo por miedo a los efectos secundarios de dicho fármaco por lo que se decidió seguimiento cada seis meses con resonancia nuclear magnética (RNM) abdominal y en función de la progresión se replanteará decisión terapéutica. 
Imagen 1. TAC cerebral: Múltiples calcificaciones subependimarias en ambos ventrículos laterales (hamartomas).

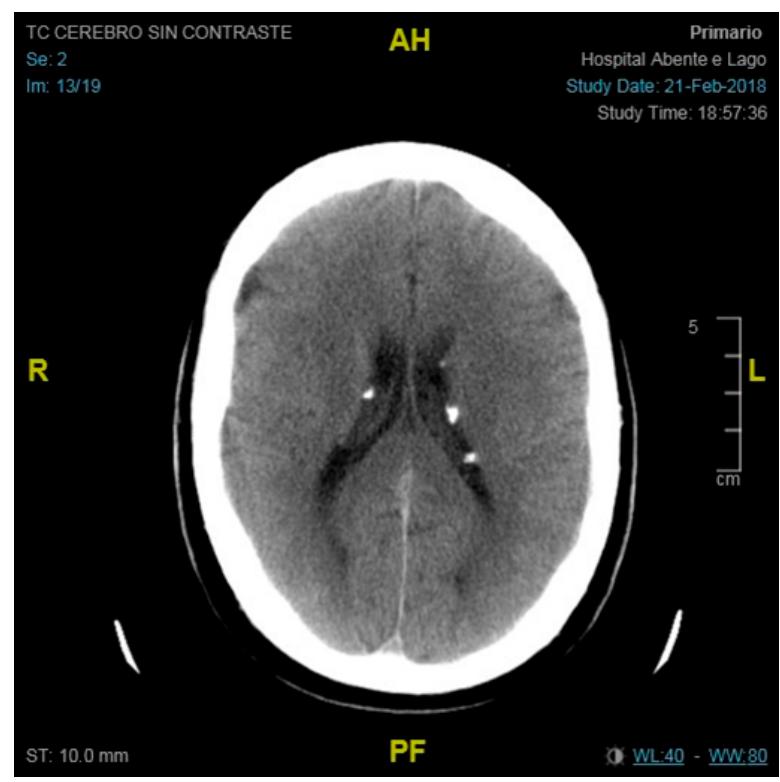

\section{DISCUSIÓN}

La ET es un síndrome neurocutáneo de herencia autosómica dominante de afectación multisistémica, caracterizado por el desarrollo de múltiples tumores benignos (hamartomas) en diferentes órganos, principalmente sistema nervioso central (SNC), ojos, corazón, pulmón, riñón, hígado y piel. También existe un mayor riesgo de desarrollar tumores malignos. El fenotipo es muy variable incluso en individuos de la misma familia y puede manifestarse tanto en el período prenatal como en la niñez, adolescencia 0 edad adultat ${ }^{1-4}$.

Se estima que la incidencia es de entre 5000 y 10000 nacidos vivos ${ }^{2,5,6}$. Se debe a una mutación en el gen TSC1 (que codifica la proteína hamartina) o TSC2 (que codifica la proteína tuberina), que forman el complejo hamartina-tuberina
Imagen 2: RNm abdominal: Angiomiolipoma renal de mayor tamaño en el riñón derecho de 3,6 cm x $4 \mathrm{~cm}$.

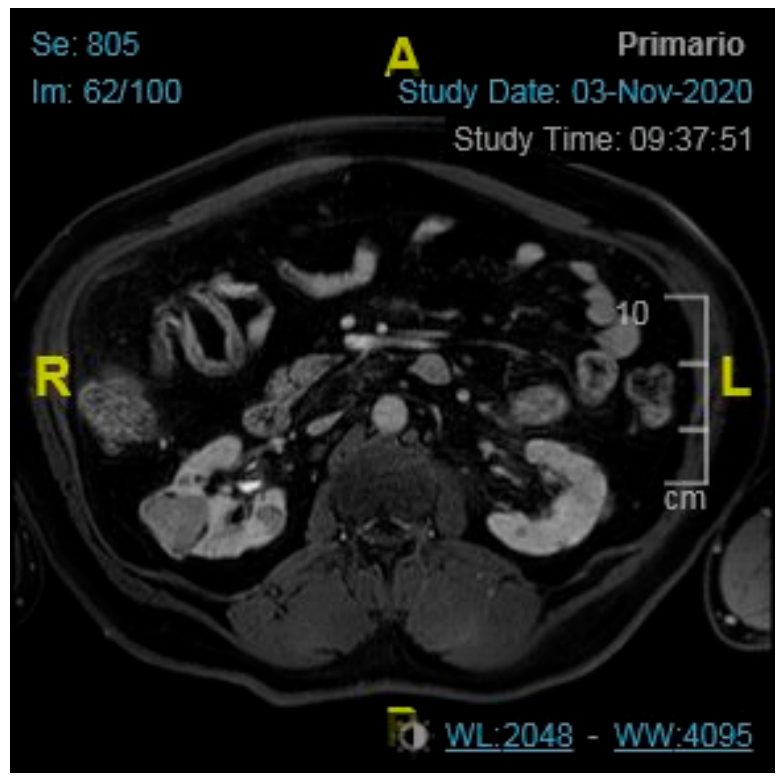

responsable de inhibir la expresión de la vía mTOR, un regulador intracelular del crecimiento y metabolismo celular. Un pequeño número de pacientes con deleción en el gen TSC2 puede asociar deleción en el gen PKD1 de poliquistosis renal. En el $80 \%$ de los casos las mutaciones son de novo?.

Prácticamente todos los pacientes presentan lesiones cutáneas características de la enfermedad, que tienden a estabilizarse después de la pubertad. Los más comunes son las manchas hipopigmentadas, los angiofibromas, las placas de Shagreen y los fibromas ungueales e intraorales. Pero solo los casos severos con mucha expresividad tienen manifestaciones en la primera infancia.
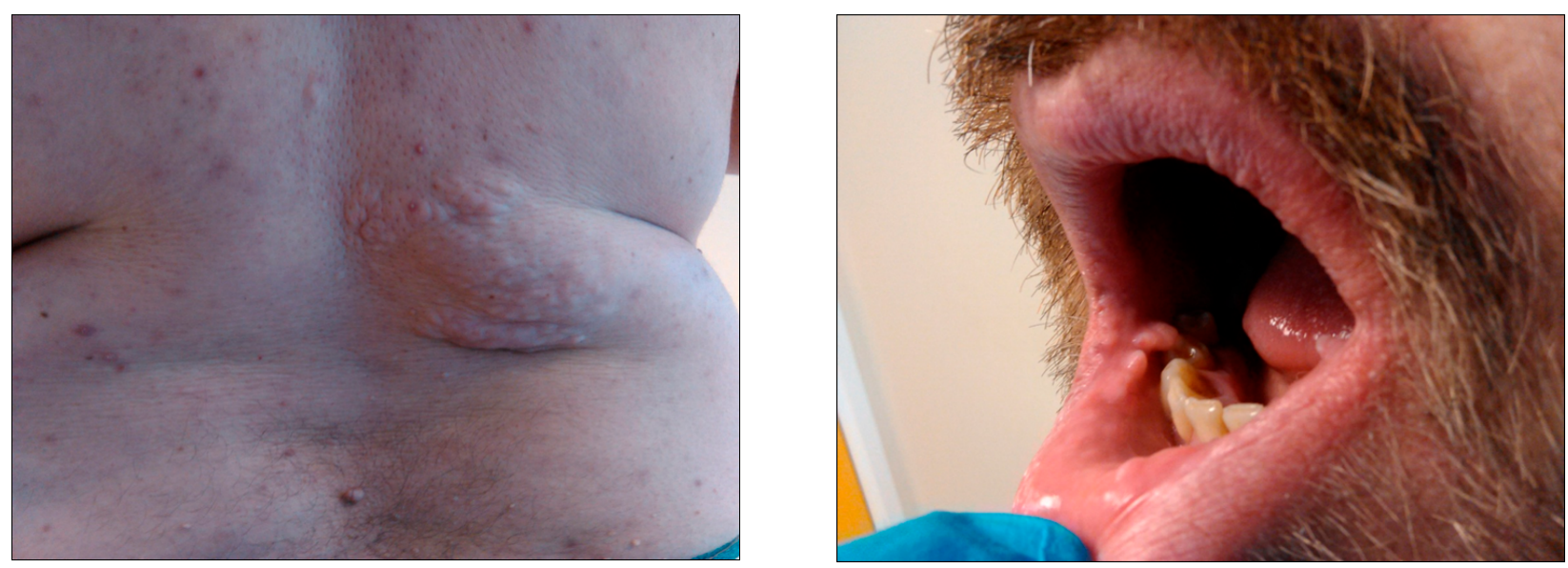
Las lesiones cerebrales típicas incluyen hamartomas glioneuronales también llamados tubérculos corticales, heterotopía de la sustancia blanca, nódulos subependimarios y tumores subependimarios de células gigantes, también conocidos como astrocitomas subependimarios de células gigantes $(\mathrm{SEGAs})^{8}$. Se ha descrito la malignidad de este último.

La mayoría de los pacientes tienen epilepsia que puede ser muy difícil de manejar, y la mitad o más, déficits cognitivos y problemas de aprendizaje. El autismo y los comportamientos parecidos al autismo son comunes en los niños con esta enfermedad ${ }^{9}$. La afectación cardíaca característica es el rabdomioma, un tumor benigno que suele ser multifocal, suele presentarse en recién nacidos y que puede detectarse intrauterino mediante ecografía. La mayoría son asintomáticos y, a menudo, presentan regresión espontánea en la infancia ${ }^{10}$.

Los angiomiolipomas son la manifestación renal más común y pueden volverse malignos. Su incidencia y tamaño aumentan con la edad y el sexo femenino y suelen objetivarse desde el primer año. Suelen ser asintomáticos pero pueden producir un efecto masa o sangrar y poner en peligro la vida del paciente. El riesgo de hemorragia es mayor en lesiones muy vascularizadas, si tienen aneurismas $>5 \mathrm{~mm}$ o durante el embarazo. También se pueden observar quistes renales y un mayor riesgo de desarrollar carcinoma de células renales, que suele ser bilateral, multifocal y en edades más tempranas. Con menor frecuencia: oncocitomas renales, enfermedades intersticiales o glomeruloesclerosis focal y segmentaria que pueden derivar en insuficiencia renal.

Algunos adultos, principalmente mujeres, desarrollan una afectación pulmonar similar a la linfangioleiomiomatosis, aunque de evolución más indolente.

Los hallazgos oftalmológicos incluyen alteraciones retinianas y no retinianas. Rara vez afectan la visión.

El diagnóstico puede realizarse mediante criterios clínicos (Tabla 1) o pruebas genéticas ${ }^{11}$. Si cumplen con los criterios clínicos de ET "definido", el estudio genéticono es necesario para confirmarlo, aunque sí permite un adecuado asesoramiento genético para futuros embarazos o identificar a familiares afectados con presentaciones más leves.

La identificación de una mutación patogénica en los genes TSC1 o TSC2 es suficiente para un diagnóstico definitivo. Sin embargo, una prueba genética negativa no excluye el diagnóstico, ya que entre el 10 y el $25 \%$ de los casos de personas con ET no se identifican estas mutaciones. Aunque existe alguna asociación (fenotipo TSC1 más leve que TSC2), una mutación determinada no predice la gravedad o el riesgo de complicaciones.

El seguimiento de estos pacientes debe ser al menos anual ${ }^{11}$. En nuestro centro se ha realizado un protocolo para su correcta valoración y seguimiento. A continuación destaco los puntos más importantes.

Desde el punto de vista neurológico se realiza un seguimiento de las crisis, ajuste de tratamiento y evaluación de trastornos neuropsiquiátricos asociados por si necesitan alguna intervención específica. La RNM cerebral sin/con contraste es la técnica de elección aunque podría ser suficiente realizar TAC con protocolo de baja radiación. Se debe realizar cada 1-3 años hasta los 25 años para rastreo de tumores subependimarios de células gigantes (SEGAs). Si no SEGAs a los 25 años, finalizar el control.

La RNM abdominal se debe realizar anualmente si existen angiomiolipomas pero puede aumentarse a 2-3 años si son menores de $1 \mathrm{~cm}$, y se realizarán cada 6 meses si miden más de $4 \mathrm{~cm}$ y se ha decidido observación, 0 si la paciente está embarazada. La frecuencia de los controles radiológicos también se aumenta si se está a tratamiento con inhibidores de la vía mTOR durante el primer año del inicio del mismo.

Si no tiene angiomiolipomas la RNM abdominal se hace cada 3 años.

La evaluación pulmonar se hace siempre en mujeres y hombres sintomáticos y también en mujeres mayores de 18 años asintomáticas ${ }^{12}$. La frecuencia de la realización del TCAR pulmonar es cada 10 años en mujeres asintomáticas y/o que tengan TCAR basal normal. Si se objetivan quistes, la frecuencia varía según la capacidad funcional medida a través de las pruebas de función respiratoria y del test de paseo. En las mujeres embarazadas, el seguimiento debe de ser estrecho sobre todo en el $3^{\circ}$ trimestre.

Este paciente posiblemente se trata de una mutación de novo dado que no refiere antecedentes familiares. En este paciente no sería estrictamente necesario la realización del estudio genético ya que cumple criterios clínicos para esclerosis tuberosa definida pero este paciente tiene un hijo con ET en edad reproductiva y su descendencia podría beneficiarse de diagnóstico preimplantacional o prenatal por lo que estaría indicada en este caso ${ }^{13}$. También es obligado el estudio de los otros hijos, ya que el fenotipo de presentación es muy variable incluso dentro de una misma familia.

Con respecto al seguimiento, ya no estaría indicado realizar RNM cerebral de control dado que ya ha superado los 25 años y no ha desarrollado SEGAs y la RNM abdominal debe hacerse cada 6 meses para vigilar el crecimiento de los angiomiolipomas y especialmente el de mayor tamaño $(4 \mathrm{~cm})$ y detectar precozmente, signos que sugieran malignidad como necrosis o calcificación o la aparición de microaneurismas que conllevan mayor riesgo de sangrado.

Con respecto al tratamiento, algunos autores optan por seguimiento cada 6 meses en angiomiolipomas entre 4-8 cm, pero la mayoría de los expertos recomiendan tratamiento ${ }^{14}$.

En este caso habría dos opciones, la embolización arterial selectiva profiláctica que sería, para la mayoría de los autores, el tratamiento de elección y se realiza en angiomiolipomas de más de $4 \mathrm{~cm}$ sobre todo si están muy vascularizados 0 presentan microaneurismas intratumorales de $\geq 5 \mathrm{~mm}$ ya que tienen más riesgo de sangrado, o tratamiento con everolimus, fármaco inhibidor de la vía mTOR, que ha demostrado reducir el volumen del tumor y disminuir el riesgo de progresión ${ }^{15}$. 
En este caso se decidió tratamiento con Everolimus pero de momento no se ha iniciado, por decisión del paciente, debido a sus frecuentes reacciones adversas: estomatitis, diarrea, fiebre, nasofaringitis, neumonitis no infecciosa y más riesgo infecciones, decidiendo seguimiento más estrecho.

\section{BIBLIOGRAFÍA}

1. Crino PB, Nathanson $\mathrm{KL}$, Henske EP. The tuberous sclerosis complex. N Engl J Med. 2006 Sep;355(13):1345-56.

2. Curatolo P, Bombardieri R, Jozwiak S. Tuberous sclerosis. Lancet. 2008 Aug;372(9639):657-68.

3. Schwartz RA, Fernández G, Kotulska $\mathrm{K}$ et al. Tuberous sclerosis complex: advances in diagnosis, genetics, and management. J Am Acad Dermatol. 2007 Aug;57(2):189.

4. Northrup H, Wheless JW, Bertin TK et al. Variability of expression in tuberous sclerosis. J Med Genet. 1993 Jan;30(1):41.

5. Hallett L, Foster T, Liu Z, Blieden M, Valentim J. Burden of disease and unmet needs in tuberous sclerosis complex with neurological manifestations: systematic review. Curr Med Res Opin. 2011 Aug;27(8):1571-83.

6. Hong $\mathrm{CH}$, Tu HP, Lin JR, Lee CH. An estimation of the incidence of tuberous sclerosis complex in a nationwide retrospective cohort study (1997-2010). Br J Dermatol. 2016 Jun;174(6):1282-9.

7. Au KS, Williams AT, Roach ES et al. Genotype/phenotype correlation in 325 individuals referred for a diagnosis of tuberous sclerosis complex in the United States. LGenet Med. 2007 Feb;9(2):88.

8. Mizuguchi M, Takashima S Neuropathology of tuberous sclerosis. Brain Dev. 2001 Nov;23(7):508
9. Curatolo P, Moavero R, de Vries PJ. Neurological and neuropsychiatric aspects of tuberous sclerosis complex. Lancet Neurol. $2015 \mathrm{Jul} ; 14(7): 733-45$

10. Hinton RB, Prakash A, Romp RL, Krueger DA, Knilans TK; International Tuberous Sclerosis Consensus Group. Cardiovascular manifestations of tuberous sclerosis complex and summary of the revised diagnostic criteria and surveillance and management recommendations from the International Tuberous Sclerosis Consensus Group. J Am Heart Assoc. 2014 Nov;3(6):e001493.

11. Northrup $H$, Krueger DA, International Tuberous Sclerosis Complex Consensus Group Tuberous sclerosis complex diagnostic criteria update: recommendations of the 2012 International Tuberous Sclerosis Complex Consensus Conference. Pediatr Neurol. 2013 Oct; $49(4): 243$.

12. Cudzilo CJ, Szczesniak RD, Brody AS, Rattan MS, Krueger DA, Bissler JJ, et al. Lymphangioleiomyomatosis screening in women with tuberous sclerosis. Chest. 2013 Aug;144(2):578-585.

13. Simpson JL. Preimplantation genetic diagnosis at 20 years. Prenat Diagn. 2010 Jul;30(7):682.

14. Kingswood JC, Bissler JJ, Budde K, Hulbert J, Guay-Woodford L, Sampson JR, Sauter M, Cox J, Patel U, Elmslie F, Anderson C, Zonnenberg BA. Review of the Tuberous Sclerosis Renal Guidelines from the 2012 Consensus Conference: Current Data and Future Study. Nephron. 2016 Aug;134(2):51-58.

15. Bissler JJ, Kingswood JC, Radzikowska E et al..Everolimus for angiomyolipoma associated with tuberous sclerosis complex or sporadic lymphangioleiomyomatosis (EXIST-2): a multicentre, randomised, double-blind, placebo-controlled trial. Lancet 2013 Mar; 381(9869):817. 